\title{
Occult Elbow Dislocation with Brachial Artery Injury
}

\section{Kazuo Ikeda* and Naoki Osamura}

Department of Orthopaedic Surgery, Kanazawa Medical Center, Japan

Keywords: Brachial artery; Surgery; Elbow dislocation

\section{Introduction}

Although elbow dislocation is the second most frequent type of dislocation in the human body, complications in the brachial artery are rare [1]. In addition, brachial artery injury due to elbow dislocation which is not discernible during the initial examination (occult dislocation) is even more rare [2]. Since reduction of the dislocated elbow has already happened to be taken place at the scene, it is difficult to detect the elbow dislocation or understand why brachial artery occlusion has occurred when there is no damage to the bone or elbow joint structures. In this paper, we examine a case of brachial artery injury with occult elbow dislocation.

\section{Case Description}

A 40-year-old male fell off a 1.5 meter ladder. He did not know that he had dislocated his elbow, but immediately consulted our hospital because his left hand was cold and he was experiencing severe forearm pain. His vital sign was stable. There was subcutaneous bleeding at the elbow joint, swelling of the forearm (Figure 1), and no pulse in the radial artery. Although he could not move his arm because of pain, passive assisted ROM (range of motion) of the elbow was 80 degrees of flexion and an extension deficit of 20 degrees. There was no instability at the elbow joint. While X-ray findings showed no damage at the elbow joint, contrast CT imaging detected an obstruction of the brachial artery 6 $\mathrm{cm}$ above the elbow joint (Figure 2). The elbow dislocation however



Figure 1: There is subcutaneous bleeding at the elbow joint and swelling in the forearm. Pulsation of the radial artery has disappeared.

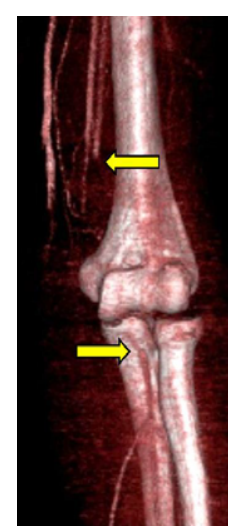

Figure 2: Contrast CT imaging shows an obstruction of the brachial artery 6 $\mathrm{cm}$ above the elbow joint level (arrow). Concomitant veins are also seen. The distal artery appears at the coronoid process from the collateral flow (arrow). The CT shows no damage in the bone or elbow joint structure. was only discovered when general anaesthesia was administered in the operating room (Figure 3). The ulnar collateral ligament was immediately sutured using a suture anchor and the brachial artery was reconstructed by an end-to-end suture with a $3 \mathrm{~cm}$ resection of the damaged area. Directly after surgery, the anastomosis of the artery was strong enough to endure the tension of elbow extension and an orthosis of 30 degrees elbow flexion was applied. One week after surgery, a joint apparatus which restricted lateral instability was applied in order to allow early motion of the elbow joint. The patient did not experience any complications such as intermittent claudication or gangrene of the hand and returned to his electric maintenance job of his original job after 2 months. The final ROM of the elbow was 140 degrees of flexion and an extension deficit of 20 degrees.

\section{Discussion}

Acute elbow dislocation with arterial rupture is rare. Ayel JE et al. reported 835 elbow dislocations in 6 years and 9 cases $(1.0 \%)$ of arterial rupture [1]. Because of the length of a usually brachial arterial injury, cephalic or concomitant vein graft is usually necessary after re-sectioning the damaged part of the artery [1,3-5]. Ayel JE et al. reported 8 cases out of 9 [1] and Platz A et al. reported 3 cases out of 4 [5] that needed vein grafts. In our case, since the length of the damaged artery was only $3 \mathrm{~cm}$, it was short enough to be sutured by the end-to-end technique. Since reduction of the elbow dislocation had already happened to be taken place at the scene, we could not recognize the elbow dislocation in the initial examination. The elbow joint was

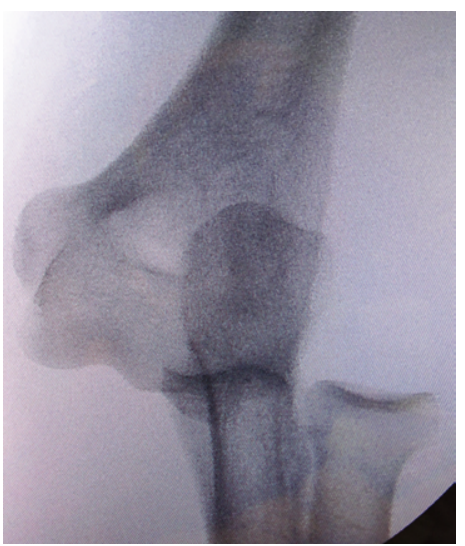

Figure 3: The elbow dislocation is discovered when general anaesthesia is administered in the operating room.

*Corresponding author: Kazuo Ikeda, Department of Orthopaedic Surgery, Kanazawa Medical Center, 1-1 Shimoishibikimachi, Kanazawa, Japan, Tel: 076-262-5931; E-mail: ikeikemed@yahoo.co.jp

Received July 08, 2014; Accepted September 19, 2014; Published September 25,2014

Citation: Ikeda K, Osamura N (2014) Occult Elbow Dislocation with Brachial Artery Injury. J Clin Case Rep 4: 420. doi:10.4172/2165-7920.1000420

Copyright: ( $2014 \mathrm{lkeda} \mathrm{K}$, et al. This is an open-access article distributed under the terms of the Creative Commons Attribution License, which permits unrestricted use, distribution, and reproduction in any medium, provided the original author and source are credited. 
stable after reduction because the patient was muscular. Although we could not understand the cause of the brachial artery occlusion at first, the elbow dislocation was discovered when general anaesthesia was administered in the operating room. Hence, elbow dislocation must be considered the differential diagnosis when there is a brachial artery occlusion without any damage to the bone or elbow joint structure.

Brachial artery occlusion is usually diagnosed by a classic catheter angiogram $[4,6]$. However, it might take two or more hours to prepare the angiogram room and call the radiologist. Since, the quality of CT imaging has improved; contrast CT imaging can reveal an obstruction point more quickly and easily than an angiography. Therefore, we recommend contrast CT imaging in order to diagnosis a traumatic obstruction of the brachial artery.

If it takes more than several hours to reconstruct the brachial artery, there is a danger of a compartment syndrome in the forearm [6]. But in our case, since the arterial reconstruction was performed quickly, we could prevent forearm compartment syndrome. Quick diagnosis and arterial reconstruction is important in these cases [6].

After we repaired the ulnar collateral ligament, the final ROM of the elbow was a flexion of 140 degrees and an extension deficit of 20 degrees. In addition, a joint apparatus which restricted lateral instability was applied in order to early motion of the elbow joint. Platz A et al. also recommended suturing of the ulnar collateral ligament and early motion of the elbow joint [5]. They reported that 3 out of 4 cases of immediate ulnar collateral ligament reconstruction showed good results as the average ROM of the elbow was 128 degrees of flexion (120-135) and the extension deficit was 7.5 degrees (0-15). Hence, quick vascular repair, immediate stability, and early motion resulted in a good clinical result for the occult elbow dislocation.

\section{References}

1. Ayel JE, Bonnevialle N, Lafosse JM, Pidhorz L, Al Homsy M, et al. (2009) Acute elbow dislocation with arterial rupture. Analysis of nine cases. Orthop Traumatol Surg Res 95: 343-351.

2. McMurtry IA, Bhullar TJ (2000) Occult elbow dislocation: a rare cause of upper limb arterial insufficiency. J R Army Med Corps 146: 230-231.

3. Thomas LG, Williams DT (2012) Simple posterior elbow dislocation and brachial artery transection. J R Army Med Corps 158: 50-52.

4. Jain K, Shashi Kumar Y, Mruthyunjaya, Ravishankar R, Nair AV (2010) Posterior dislocation of elbow with brachial artery injury. J Emerg Trauma Shock 3: 308.

5. Platz A, Heinzelmann M, Ertel W, Trentz O (1999) Posterior elbow dislocation with associated vascular injury after blunt trauma. J Trauma 46: 948-950.

6. Carter SJ, Germann CA, Dacus AA, Sweeney TW, Perron AD (2010) Orthopedic pitfalls in the ED: neurovascular injury associated with posterior elbow dislocations. Am J Emerg Med 28: 960-965. 